\title{
Study on the Strength of the LIPEV Frame Based on ANSYS
}

\author{
Meishi zhou ${ }^{1, a}$, Tiezhu Zhang ${ }^{2, b^{*}}$, Huaixian Yin ${ }^{3, c}$, Hongxin Zhang ${ }^{4, d}$,
}

\author{
Gaojun Liu ${ }^{5, e}$ \\ ${ }^{1}$ Mechanical and Electronic Engineering College, QingDao University, Qingdao 266071, China \\ ${ }^{2}$ Scientific Research Office, QingDao University, Qingdao 266071, China \\ a1015988605@qq.com, byhx6279010@163.com, ${ }^{\mathrm{a} q d z h a n g t z @ 163 . c o m,{ }^{d} q d u z h x @ 126 . c o m,}$ \\ eqdlgj@163.com
}

Key words: load isolated pure electric vehicle(LIPEV); frame; ANSYS; strength analysis

Abstract: Aiming at the frame strength of the load isolated pure electric vehicle (LIPEV) which is modified by a micro truck, the three-dimensional model of the frame was established based on UG. The finite element analysis model of the frame was established based on ANSYS. The load before modified and the load after modified was carried out on the frame to solve the maximum equivalent stress of the frame. The value of the maximum equivalent stress with full load bending condition before modified is $2.57 \mathrm{Mpa}$ while the value after modified is $2.57 \mathrm{MPa}$. The value of the maximum equivalent stress with floating condition before modified is $43.8 \mathrm{MPa}$ while the value after modified is $43.7 \mathrm{MPa}$. The results show that although the modification of the LIPEV has changed the load distribution of the frame, the frame can meet the strength requirement of the LIPEV frame, which can provide the theoretical basis for the modification of other electric vehicles.

\section{Introduction}

The shortage of petroleum resources is a worldwide problem, so the key point to develop the automobile is to reduce its dependence on oil. Therefore, the development of hybrid vehicles and pure electric vehicles becomes an inevitable trend [1]. At present, most pure electric vehicles are modified by traditional vehicles. The strength of the frame should be verified, because the load is different between the original engine vehicle and the modified vehicle called as the load isolated pure electric vehicle(LIPEV). A large number of domestic and foreign scholars have carried out a lot of research on the reliability of the modified frame. For example, Jinxia Sun do static analysis by finite element technique for fuel cell vehicle's frame[2]. In this paper, the three-dimensional model of the frame was established based on UG. The finite element analysis model of the frame was established based on ANSYS. Before and after modified load were carried out on the frame to solve the maximum equivalent stress, which is to verify if the strength of the frame could meet requirement after modification. The micro truck is shown as Figure 1. 


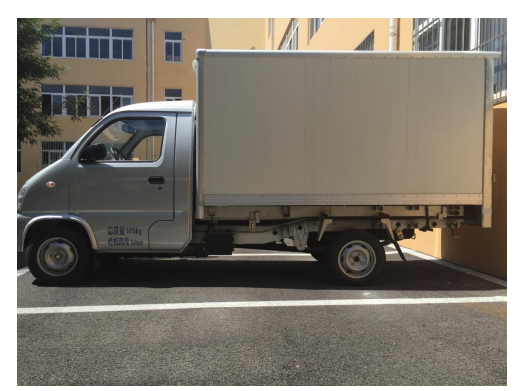

Fig. 1 the figure of micro truck

\section{Data storage}

There are two working conditions which is the most common condition and the most danger condition that have been considered in the statics analysis of the frame: full load bending condition, one wheel left floating condition.

full load bending condition mainly simulates the condition that the vehicle in the full load was driven in a good road at the speed of $50 \mathrm{~km} / \mathrm{h}$. This condition is the most common condition when the vehicle was driven.

Due to the effect of the road surface roughness, the vehicle in the driving process is subjected to torsional load, so that the frame is subjected to torque. Consulting the relevant literature, the truck frame suffered the most severe torsion condition is generally its low speed through the rough pavement [3], and this condition is the most dangerous working condition of the frame. So it is very important to analyze the static strength of this condition.

The length of the frame is $4610 \mathrm{~mm}$ and the width of the frame is $1060 \mathrm{~mm}$. The material of the frame is Q345 steel. Elastic modulus is $2.06 \times 10^{5} \mathrm{MPa}$. Poisson's ratio is 0.28 . The density of the frame material is $7850 \mathrm{~kg} / \mathrm{m}^{3}$. The yield limit is $345 \mathrm{MPa}$. Full quality of the frame is $97.26 \mathrm{~kg}$.

\section{The finite element analysis of the frame}

Model simplified. First of all, the frame of the 3D model was established based on UG, as shown in figure 2, and imported it into the ANSYS to ensure static analysis from multiple conditions.

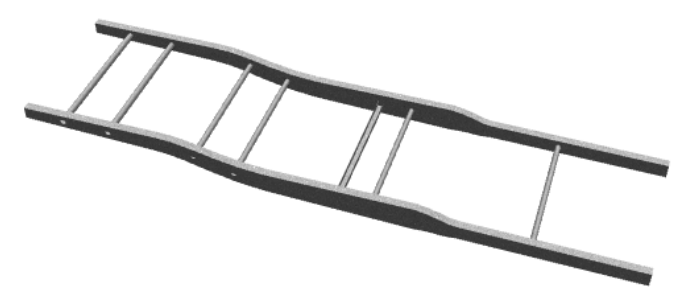

Fig. 2 the three-dimensional model of the micro truck

Considering the model was built based on UG, and the shape of frame was complex, the element type solid187 was chosen to make sure the calculation accuracy[4]. Because the solid187 element is one of the most element that can express the information of actual parts. It can not only express the quality, inertia, material and other characteristics of the parts, but also be used to approximate the real geometry from the point of view of space, especially the geometric model which can reflect all the geometric changes[5]. The element size was set to $0.01 \mathrm{~mm}$, and the finite element model of the frame has 94946 nodes and 285459 elements.

Load simplified. The driving scheme of the original micro truck was FR type while the driving scheme of the load isolated pure electric vehicle was FR type. What is more, the load 
isolated pure electric vehicle added the battery box which weight $300 \mathrm{~kg}$ and the engine was replaced by a smaller one compared to the original micro truck. The prototype vehicle model is established to extract dynamic loads, especially the impact loads of short time rapid actions between each key connecting part and the frame. At the same time, the frame finite element model was established to ensure strength and stiffness analysis with dynamic loads as boundary conditions. the loads of the original vehicle frame is shown as table1. the loads of the modified vehicle frame is shown as table2.

Tab.1 the load of the original vehicle frame

\begin{tabular}{cccc}
\hline Main part and assembly & Mass $/ \mathrm{kg}$ & Load type & Value of dynamic load/N \\
\hline Cab and drivers & 400 & Uniform force & $8.7312 \times 10^{3}$ \\
Cargo capacity & 930 & Uniform force & $2.0234 \times 10^{4}$ \\
engine and transmission & 300 & Uniform force & $7.836 \times 10^{3}$ \\
\hline
\end{tabular}

Tab. 2 the load of the modified vehicle frame

\begin{tabular}{cccc}
\hline Main part and assembly & Mass $/ \mathrm{kg}$ & Load type & Value of dynamic load/N \\
\hline Cab and drivers & 400 & Uniform force & $8.7312 \times 10^{3}$ \\
Cargo capacity & 930 & Uniform force & $2.0234 \times 10^{4}$ \\
Battery box & 300 & Uniform force & $7.836 \times 10^{3}$ \\
engine and motor & 160 & Uniform force & $4.0768 \times 10^{3}$ \\
\hline
\end{tabular}

The material of the spring is $55 \mathrm{Si} 2 \mathrm{Mn}$. The elastic modulus is $2.07 \times 10^{5} \mathrm{MPa}$. Poisson's ratio is 0.27 . The density of the spring material is $7730 \mathrm{~kg} / \mathrm{m}^{3}$. The elastic element of the front suspension is spiral spring while the elastic element of the rear suspension is leaf spring. Because there is no leaf spring in the software ANSYS, the method of the three degrees of freedom model of leaf Spring was adopt. In this method, one leaf spring is equivalent to two groups' three-direction spring that along with UX, UY, UZ. the reaction force of the three springs generated by the frame can withstand the lateral force, longitudinal force. Considered all the equivalent model of leaf spring, the springs can also withstand the rolling moment, pitching moment, and yawing moment. Hence, this equivalent model is close to the actual leaf spring working conditions. Connecting the common node of three direction spiral spring elements with lug support multiple nodes of the frame by the form of RBE3, as shown in figure 3. Refer to other papers, the vertical stiffness of the leaf spring is $7.875 \times 10^{5} \mathrm{~N} / \mathrm{m}[6]$.

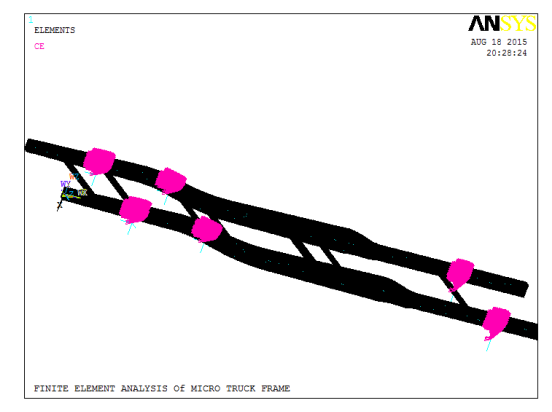

Fig. 3 the finite element model of the frame

The boundary conditions are determined according to the driving condition analysis and the relevant information. The full load bending condition mainly simulate vehicle under full load 
condition, high-speed on a good road condition. The constraint of the right front leaf spring is $\mathrm{x}, \mathrm{y}, \mathrm{z}$ directions of freedom; the constraint of the left front leaf spring is $\mathrm{x}, \mathrm{z}$ directions of freedom; the constraint of the right rear leaf spring is $y, z$ directions of freedom; the constraint of the left rear leaf spring is $\mathrm{z}$ direction of freedom.

The constraint of one left floating condition is that only two sets left rear springs were released under the condition of full load bending.

Static analysis of frame structure. The static analysis of the frame structure includes two aspects: rigidity analysis and strength analysis. In finite element analysis, the static analysis of the control equation is as follows:

$$
\{K\} U\}=\{F\} .
$$

In this formula, $\{K\}$ is the structural stiffness matrix; $\{U\}$ is the displacement vector; $\{F\}$ is the load vector [7].

\section{The strength compared between the original micro truck and the modified one}

The ability of metal material to resist permanent deformation and fracture under the action of an external force is called the strength. According to the fourth strength theory of material mechanics, the stress was chosen to evaluate the strength. Stress can be expressed as:

$$
\sigma_{r 4}=\sqrt{\frac{\left(\sigma_{1}-\sigma_{2}\right)^{2}+\left(\sigma_{2}-\sigma_{3}\right)^{2}+\left(\sigma_{3}-\sigma_{1}\right)^{2}}{2}} .
$$

$\sigma_{1}$ is the first main stress; $\sigma_{2}$ is the second main stress; $\sigma_{3}$ is the third main stress; $\sigma_{r 4}$ is the fourth equivalent stress. When $\sigma_{r 4} \leq[\sigma]$, the material is meet with the strength requirements[8].

The frame equivalent stress distribution of the full load bending condition with the original load as boundary condition is shown as fig.4. The maximum equivalent stress is $2.57 \mathrm{MPa}$. The frame equivalent stress distribution of the full load bending condition with the LIPEV load as boundary condition is shown as fig.5. The maximum stress is $2.57 \mathrm{MPa}$ which was far less than the material allowable stress after modification. The frame meets the strength requirement and the load layout of the frame is reasonable after modification .

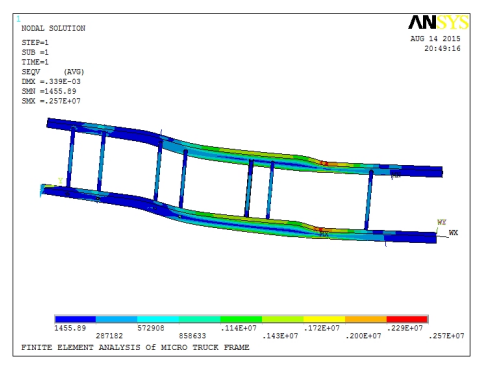

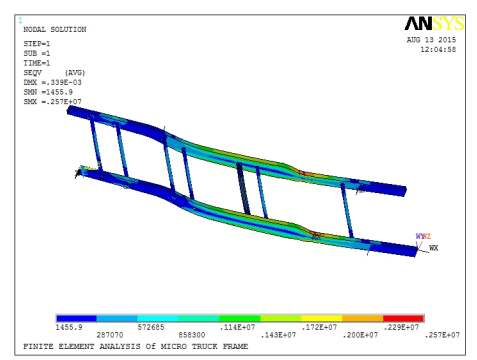

Fig.4 von mises of the original load with full Fig.5von mises of the LIPEV load with full

The frame equivalent stress distribution of the one left floating condition with the original load as boundary condition is shown as fig.6. The maximum equivalent stress is $43.8 \mathrm{MPa}$. The frame equivalent stress distribution of the frame with the latter load as boundary condition is shown as fig.7. The maximum stress is $43.7 \mathrm{MPa}$ which was far less than the material allowable stress after modification. The frame meets the strength requirement and the load layout is reasonable after modification of the frame. 


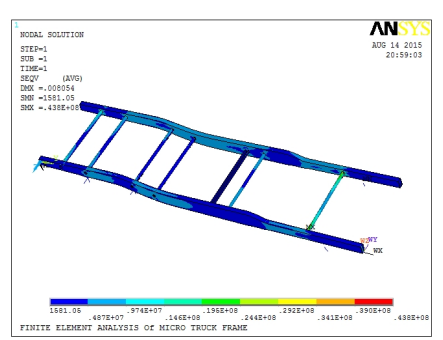

Fig.6 von mises of the original load with floating

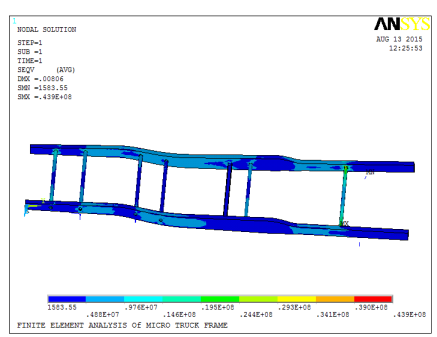

Fig. 7 von mises of the LIPEV load with floating

\section{Conclusion}

The three-dimensional model of the frame was established based on UG. The finite element analysis model of the frame was established based on ANSYS. The load before modified and the load after modified were carried out on the frame to solve the maximum equivalent stress of the frame. The results show that although the electric vehicle modification has changed the load distribution of the frame, the frame can meet the strength requirement of the electric vehicle frame, which can provide the theoretical basis for the modification of other electric vehicles.

\section{Acknowledgements}

The authors gratefully acknowledge the financial support from the Funded by the National High-tech R\&D Program(863Program) of China(No.2014AA052303), Provincial Shandong Province Foundation of China (No. 2014ZZCX05501 and No. 2014GGX103044). Cultivation of New and Strategical Industries of Qingdao, Shandong, China(14-8-1-2-gx).

\section{Reference}

[1] Hanming Chen. Mechanical \& Electrical Engineering Technology. 5 (2001) 17-20. In Chinese [2] Jinxia Sun. Shanghai Auto. 6 (2006) 36-38. In Chinese

[3] Xinwen Chai, Shiwen Yang. Mechanical Management And Development. 2 (2010) 51-52. In Chinese

[4] Moaveni, Saeed. Pearson Prentice Hall, Pearson Education International, 2003.

[5] Zhucheng Wang. Tsinghua University Press. Beijing. 2009. In Chinese

[6] Anhe Yu, Liangjin Gui, Zijie Fan. Automobile Technology. 2 (2007) 26-30. In Chinese

[7] Hongxin Zhang, Huaimin Wang ed. Machinery Industry Press, Beijing, 2013. In Chinese

[8] Dabin Liu, Wenba Han, Binqing Cai. Engineering Science. 9 (2010) 44-52. In Chinese 